\title{
A New Method for the Estimation of Bearing Health State and Remaining Useful Life Based on the Moving Average Cross- Correlation of Power Spectral Density
}

Lang $\mathrm{Xu}^{1 *}$, Paolo Pennacchi ${ }^{1}$ and Steven Chatterton ${ }^{1}$

1. Dept. of Mechanical Engineering, Politecnico di Milano, Via G. La Masa 1, 20156 Milan, Italy; lang.xu@polimi.it; paolo.pennacchi@polimi.it; steven.chatterton@polimi.it;

\begin{abstract}
In the broad framework of condition-based maintenance, the final objective of bearing condition monitoring is to evaluate the health state and to estimate the remaining useful life of the bearings. The latter is a particularly challenging task, considering that remaining useful life is inextricably linked to a projection of what will happen in the future. Often, health indices, whose reliability relies on their effectiveness and consistency, are used for bearing condition monitoring. Most of the existing health indices pursue the property of monotonicity, but generally there are no obvious boundaries between the different health states of the bearings. Hence, it is quite difficult to give an objective and independent estimation of the health states, especially in real applications under different operating conditions and in the presence of noise. Furthermore, it is also difficult to set the failure threshold for a given health index when it is employed in different applications. In this paper, a new health index called 'MAC ${ }^{2} \mathrm{PSD}^{2}$ ' is proposed, based on the moving-average cross-correlation $\left(\mathrm{MAC}^{2}\right)$ of the power spectral density (PSD) of the vibration signals. An interesting property of MAC ${ }^{2} P S D$ is its capability to track the health condition and to discriminate clearly between the different health states. As shown in the paper, the MAC PSD can also be used to estimate the remaining useful life by using its values during the defect-propagation phase. The effectiveness of MAC ${ }^{2} P S D$ is shown by means of two different cases of bearing run-to-failure experimental data, from two different test rigs. Additionally, the capability to avoid false positives is evaluated by means of bearing vibration data measured on a locomotive in commercial service.
\end{abstract}

Keywords: bearing condition monitoring, remaining useful life, power spectral density, health index, health state, moving average cross-correlation coefficient.

\section{Introduction}

The demand for safety, reliability and economic efficiency of mechanical systems is growing with time, and increasing attention is being paid to it. In particular, safety is a prerequisite for mechanical systems designed to transport people, such as vehicles, airplanes and trains, because failures may cause catastrophic accidents. Furthermore, the maintenance cost is another focus of companies running those assets, and the condition-based maintenance approach is not yet fully exploited, mainly due to the lack of reliable estimation of the remaining useful life (RUL) of the components. As one kind of critical component in rotating machinery, rolling element bearings (REBs) may fail before their designed end of life because of out-of-design operating conditions, lubrication problems, improper handling and mounting, etc. (1). REB failures have become one of the most common causes of rotating machine breakdowns (2). The purpose of bearing condition monitoring $(\mathrm{CM})$ is to monitor and to assess the health state of bearings, then possibly to estimate the RUL of bearings, which makes it possible to intervene before machine failure and schedule maintenance in order to decrease safety risks and maintenance cost. Therefore, bearing CM is of great importance not only for guaranteeing the safe operation of a mechanical system but also for improving the market competitiveness of the companies running them.

In the last few years, an increasing number of researchers have focused on the field of bearing CM and obtained many useful outcomes (3-5). The classification of CM methods varies from the 
perspective of the researchers, but the classification of model-driven methods and data-driven methods is widely adopted. Generally, model-driven methods establish a sophisticated mathematical model according to the details of the mechanical system and often are able to estimate the RUL accurately (5). However, model-driven methods need an in-depth understanding of the mechanical system, which is often non-feasible for complex mechanical systems in actual cases. The data-driven method is the predominant approach for CM of mechanical systems and has attracted most of the attention. Data-driven methods utilize the data collected by the data acquisition system to extract some useful health indices (HIs). These HIs are then applied to monitor the health state and forecast the RUL of the machine (6). Therefore, the performance of a CM method is largely dominated by the extracted HI. As the health condition index of bearings, a good HI should be sensitive to not only the existence of the defect but also the evolution of the defect. Time-domain features, frequency-domain features, time-frequency features or the fusion of these are commonly used to construct HIs. They are usually divided into two categories, including physical HIs and virtual HIs in (3). Physical HIs have an exact physical meaning, such as RMS, kurtosis, Shannon entropy, etc. (7,8). Virtual HIs are without corresponding physical meaning, such as the cumulative features proposed in (9) or the multifeature fused feature via principal component analysis (PCA) in (10). As an affiliate function of HIs, health state (HS) division aims to divide the continuous life of bearings into several separate segments where every segment represents a health condition. Generally, the HS division is made according to the varying trends of HI, which means different HSs have different trends in HI variation. The HS division methods were simply divided into two groups, including a two-phase division and a multiphase division, in (3). El-Thalji et al. proposed an elaborate five-phase HS model based on the evolution of dynamic wear of the bearing surface in $(11,12)$. However, almost none of the existing HIs can clearly divide the HS state as did the five-phase approach. However, the most important function of $\mathrm{HI}$ is to estimate the RUL of bearings. Kurtosis was used to adaptively determine the first prediction time (FPT), and an exponential model based on RMS was proposed to predict the RUL of REBs in (8). Lei et al. made use of RMS combined with a stochastic process model and Kalman particle filtering algorithm to estimate the machine RUL in (13). Pennacchi et al. utilized the cyclic content ratio (RCC) to monitor the health condition of REBs on a regional train locomotive in $(14,15)$. Ocak et al. took advantage of the energies of the nodes decomposed by wavelet packets as features to track the health condition of bearings (16). Of course, there are many other methods of bearing RUL prediction based on machine learning or artificial intelligence, such as artificial neural network (ANN)-based methods, support vector machine (SVM)-based methods or Gaussian process regression (GPR)-based methods. (17-19). However, literature based on the similarity of the present state and the previous state of bearings is scarce. Medjaher et al. proposed an HI by evaluating the similarity of the vibration signal between the degraded bearing and the nominal bearing to estimate the RUL (20). $\mathrm{Yu}$ extracted an $\mathrm{HI}$ to track the degradation of bearings via measuring the similarity of the probability density functions of the historic hidden Markov model (HMM) with the present adapted HMM (21). Guo et al. fused some related-similarity features and several classic time-frequency features using a recurrent neural network (RNN) to create a new HI to predict the RUL of bearings (22).

Even though a considerable number of the existing data-driven methods perform well in some specific cases, there are still some problems that impede their wide application to industry. The most discussed problem is the determination of the failure threshold for the HI. At present, the vast majority of proposed HIs require the users to set a failure threshold in advance. However, in most cases, every REB in a mechanical system is different from the others, with different loads, speeds, lubrication situations, etc., which would undergo different health degradation paths. Thus, it is extremely hard to determine a threshold for each case. Another crucial reason is the consistency or universality of the HI. An HI may perform well in some cases but is useless in other cases. Inspired by the aforementioned methods, in this paper, a new health index 'MAC ${ }^{2} \mathrm{PSD}$ ' is proposed, which is the moving average cross-correlation $\left(\mathrm{MAC}^{2}\right.$ ) of the power spectrum density (PSD). PSD represents the energy distribution of a signal in the frequency domain, whereas $\mathrm{MAC}^{2}$ is the moving average cross- 
correlation used to measure the similarity of PSD of consecutive records. The rationale of this method is that the energies of fault signals and normal signals are distributed in different frequency bands in PSD, and the PSD will change with the degradation of the bearings. MAC PSD takes advantage of the variation of the PSD of the signal compared with those of the adjacent signals. Compared with other HIs, MAC ${ }^{2}$ PSD can determine a failure threshold for each case by itself when used to estimate the RUL of REBs. In addition, it can also clearly distinguish different HSs.

This paper is organized as follows: Section 2 illustrates the basic principle of MAC PSD; Section 3 explains the detail of dividing HSs based on MAC ${ }^{2} P S D$; Section 4 estimates the RUL of bearings based on MAC ${ }^{2}$ PSD via two sets of run-to-failure data of REB. Section 5 tests the performance of MAC ${ }^{2} P S D$ applied to bearing data from a regional locomotive train.

\section{Definition of the new health index MAC ${ }^{2}$ PSD}

(a)

Vibration signal records

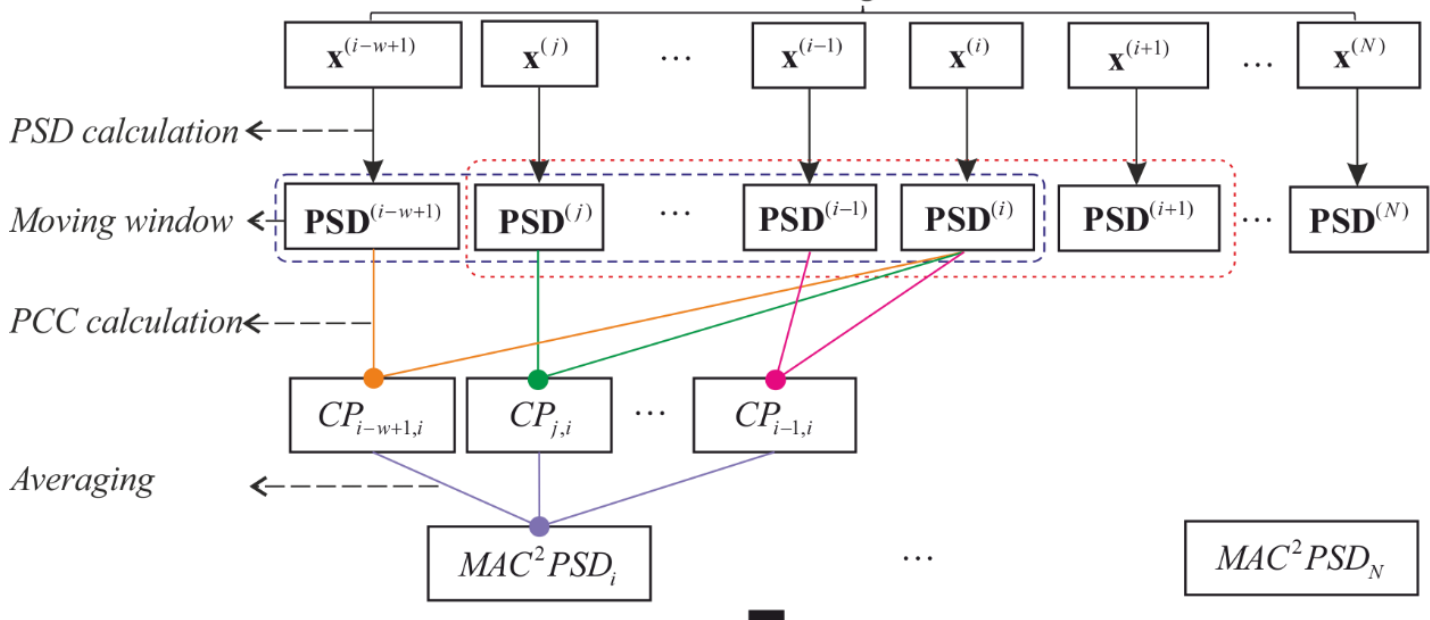

(b)

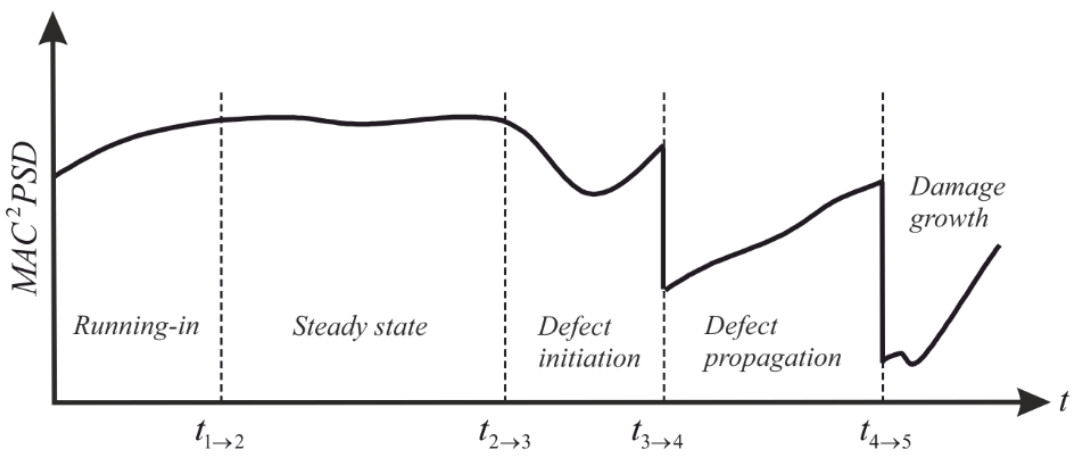

(c)

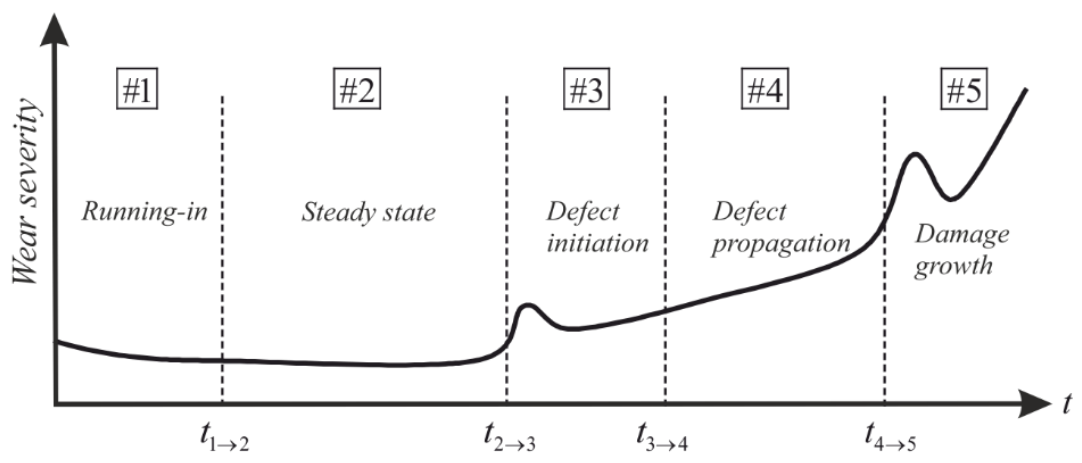


Fig. 1. (a) MAC ${ }^{2} P S D$ calculation; (b) Qualitative diagram of $M A C^{2} P S D$; (c) Wear severity during the lifetime of a bearing (adapted from $(11,12))$

Bearing wear is a complex process mainly related to fatigue (23). To classify the HS during the operational life of a bearing, El-Thalji et al. proposed a five-phase wear evolution model in $(11,12)$; see Fig. 1 (c). The phases are as follows:

1) running-in (phase \#1);

2) steady-state (phase \#2);

3) defect initiation (phase \#3);

4) defect propagation (phase \#4);

5) damage growth (phase \#5).

The changes in the HS of the bearings affect their vibrations, including the levels and the components of the signals. It is well known (24) that defects in the bearings cause a series of impacts whose intensity generally increases with the growth of the defects. The aim of all the data-driven CM methods is to track to the best of their ability what is varying in the vibration signals. Generally, the fault signals distribute in some frequency bands, which can be distinguished from the environmental noise. A reliable signal processing technique for determining the energy distribution of a signal in the frequency domain (25) is the PSD. If the frequency spectrum of the signal is compared with the PSD, the latter is more effective in highlighting the components related to the defects because the autocorrelation of the signal, employed in the algorithm for PSD calculation, is able to denoise some of the white noise (26). Therefore, the use of PSD and its variations to monitor the wear of the bearings, and their HS is rational and practicable.

Let us suppose a sequence of $N$ records is collected with a constant time interval (for instance 1 day or 1 hour) or constant cycle interval throughout the lifetime of a bearing, similar to that displayed in Fig. 2. Each record is composed of $L$ samples, i.e., $\mathbf{x}^{(i)}=\left[x_{1}^{(i)}, x_{2}^{(i)}, \cdots, x_{L}^{(i)}\right]^{\mathrm{T}}$, whereas the time instant $t_{i}$ represents the acquisition time (in hours or number of cycles) of record. As an abbreviation in this paper, the term 'time instant $t_{i}$ ' represents the acquisition time of record $\mathbf{x}^{(i)}$.

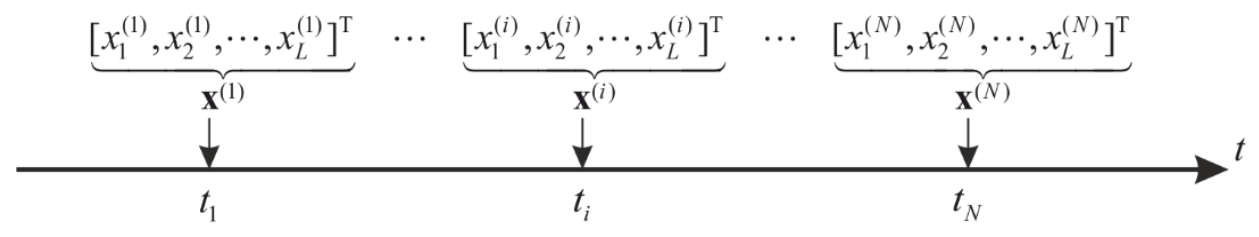

Fig. 2. Sequence of records during the lifetime of a bearing

The following hypotheses are then assumed to define the MAC ${ }^{2} P S D$ index:

a) If the HS of the bearing is stable, the PSD of a bearing vibration signal record $\mathbf{x}^{(i)}$ in a given time instant $t_{i}$ is similar to the PSD of the neighbouring signal records $\mathbf{x}^{(i-k)}$ and $\mathbf{x}^{(i+k)}$. Conversely, if the HS is changing, the PSD of a vibration signal record differs from that of the neighbouring signal records.

b) The difference among the PSD of the signal records increases with the severity of the defect.

c) The defect propagation phase is a relatively stable process.

The last hypothesis may appear as a rather strong assumption, but it is important to remember once again that the main reason for bearing damage is fatigue and that the fault growth takes millions of cycles. Therefore, from an engineering point of view, the defect propagation can be considered as a relatively stable process — or at least a not sudden one.

The algorithm for the calculation of the MAC ${ }^{2}$ PSD is shown in Fig. 1(a), and it is structured on three main steps: 
Step 1. Calculating the power spectral density of each vibration signal:

According to the Wiener-Khinchin theorem, the PSD of a signal $x(t)$ can be calculated by means of the Fourier transform of the autocorrelation function (24):

$$
P S D(f)=\mathcal{F}\left\{R_{x x}(t)\right\}
$$

while the autocorrelation function $R_{x x}(\tau)$ of a signal $x(t)$ is obtained by calculating the average value of the product of the signal $x(t)$ at time $t$ with the same signal shifted by $\tau$ (24):

$$
R_{x x}(\tau)=\mathbb{E}[x(t) x(t+\tau)]
$$

where $\mathbb{E}$ is the expected value operator.

Note that the signal $x(t)$ is considered as a function in eqs. (1) and (2), so that also $R_{x x}(\tau)$ and $P S D(f)$ are functions. For the sampled signal $\mathbf{x}^{(i)}$, the corresponding autocorrelation $\mathbf{R}_{x x}^{(i)}$ and $\mathbf{P S D}^{(i)}$ of $\mathbf{x}^{(i)}$ are symmetric vectors with a length of $2 L-1$, i.e., $\mathbf{R}_{x x}^{(i)}=\left[r_{-L}^{(i)}, r_{-L+1}^{(i)}, \cdots, r_{L}^{(i)}\right]^{\mathrm{T}}, \mathbf{P S D}^{(i)}=\left[p_{-L}^{(i)}, p_{-L+1}^{(i)}, \cdots, p_{L}^{(i)}\right]^{\mathrm{T}}$.

Step 2. Evaluating the similarity between PSDs in a moving window:

A predefined window of constant length $w$ is used for the operation of the moving average along the records and the window moves forward with a unitary step size. By considering the record $\mathbf{x}^{(i)}$ acquired at time instant $t_{i}$, there are $w$ PSDs in the window, and the similarity between the $\mathbf{P S D}^{(i)}$ of the $i^{\text {th }}$ record and each of the other $w-1$ PSDs of the remaining previous records $\mathbf{x}^{(j)},(j=i-w+1, \cdots, i-1)$ in the window (see Fig. 1(a)), is measured by means of the similarity index $C P_{j, i}$ based on the Pearson correlation coefficient (PCC) (20):

$$
C P_{j, i}=\left|\frac{\sum_{k=-L}^{L}\left(p_{k}^{(i)}-\overline{P S D^{(i)}}\right)\left(p_{k}^{(j)}-\overline{P S D^{(j)}}\right)}{\sqrt{\sum_{k=-L}^{L}\left(p_{k}^{(i)}-\overline{P S D^{(i)}}\right)^{2}} \cdot \sqrt{\sum_{k=-L}^{L}\left(p_{k}^{(j)}-\overline{P S D^{(j)}}\right)^{2}}}\right|
$$

Equation (3) can be rewritten in a compact form as:

$$
C P_{j, i}=\frac{\left.\mid \mathbf{P S D}^{(i)}-\overline{P S D^{(i)}}\right)^{T}\left(\mathbf{P S D}^{(j)}-\overline{P S D^{(j)}}\right) \mid}{\left\|\mathbf{P S D}^{(i)}-\overline{P S D^{(i)}}\right\|\left\|\mathbf{P S D}^{(j)}-\overline{P S D^{(j)}}\right\|}
$$

where $i=w, w+1, \cdots, N$, for each time instant $t_{i}, j=i-w+1, \cdots, i-1, \overline{P S D^{(i)}}=\mathbb{E}\left(\mathbf{P S D}^{(i)}\right)$ and $\overline{P S D^{(j)}}=\mathbb{E}\left(\mathbf{P S D}^{(j)}\right)$ are the mean values of $\mathbf{P S D}{ }^{(i)}$ and $\mathbf{P S D}{ }^{(j)}$ respectively. For the initial time instants $2 \leq t_{i} \leq w-1$, the window size can be assumed equal to $i$.

Step 3. Obtaining the MAC ${ }^{2} \mathrm{PSD}$ for every time instant:

For each time instant, such as time instant $t_{i}, w-1$ similarity indices $C P s$ are obtained in the window. Finally, the average value of these $C P S$ in the window allows the $\operatorname{MAC}^{2} \operatorname{PSD}_{i}$ at time instant $t_{i}$ to be obtained:

$$
M A C^{2} P S D_{i}=\frac{1}{w-1} \sum_{j=i-w+1}^{i-1} C P_{j, i}
$$

where $i=2,3, \cdots, N$.

In many actual cases, the operating conditions (rotational speed, loads, temperatures) vary during the lifetime of a bearing (27), e.g., for bearings used in trains, cars (28), mining machines (29) or wind turbines (30-32). In these cases, it is almost impossible to obtain several baseline signals (such as those considered in (20)) for every operating condition in real time. However, for the definition of the new index MAC ${ }^{2} \mathrm{PSD}$, no specific baseline is considered at all. 
The qualitative diagram of the MAC ${ }^{2} \mathrm{PSD}$ for a bearing during its lifetime is shown in Fig. 1 (b). Taking into account the definition of MAC ${ }^{2} \mathrm{PSD}$, one can expect the following:

1) a certain trend during the running-in phase: the vibration signal record $\mathbf{x}^{(i)}$ at time $t_{i}$ is slightly different from the previous ones. In view of bearing topology, this corresponds to the smoothing of surface roughness;

2) a rather stable value during the steady-state phase: the vibration signal record $\mathbf{x}^{(i)}$ at time $t_{i}$ is not very different from the previous ones. In view of bearing topology, this corresponds to uniform lubricant film and contact mechanics;

3) a variable trend during the defect initiation phase: the vibration signal record $\mathbf{x}^{(i)}$ at time $t_{i}$ is rather different from previous ones. In view of bearing topology, this corresponds to the start of surface denting;

4) a sudden change at the beginning of the defect propagation phase followed by an evident trend: the vibration signal record $\mathbf{x}^{(i)}$ at time $t_{i}$ changes strongly from the previous phase and it is also changing during this phase. In view of bearing topology, this corresponds to microcracks opening on and under the surfaces;

5) another sudden change at the beginning of the damage growth phase followed by an evident trend: the vibration signal record $\mathbf{x}^{(i)}$ at time $t_{i}$ is changing from the previous phase, and it is also changing during this phase. In view of bearing topology, this corresponds to the detachment of relatively large material debris.

\section{Use of $\mathrm{MAC}^{2} \mathrm{PSD}$ to determine the health state of bearings during run-to-failure tests}

As stated before, bearing wear is a complex process and during the different phases the degeneration rate may vary with the severity of the damage. A good HI should distinguish between the different HSs, or at least between the last ones (phases \#4 and \#5 in Fig. 1). A counterexample is given by RMS, which is not generally deemed as a "good" HI. Beyond being strongly influenced by environmental noise, RMS generally does not show remarkable variations during the steady-state phase nor during defect initiation, while it increases rapidly only before the final failure. This negative characteristic is shared with other HIs with the property of monotonicity: it is hard to define the boundaries between different HSs. In contrast, an HI characterized by discontinuities/sharp variations, such as the MAC ${ }^{2} \mathrm{PSD}$, is a good candidate for distinguishing the HSs, especially for the last two phases.

A further reason for which a "good" HI must be able to distinguish between the last HSs of a bearing life is the case for which the calculation of the RUL starts from a given instant of time $t_{\mathrm{FPT}}$, generally defined as the first predicting time (FPT) $(33,34)$. It will be immediately understood how convenient it is to calculate the FPT before the bearing enters the last phase, i.e., that of the damage growth phase.

Two experimental cases are used to show the performance of the MAC ${ }^{2} \mathrm{PSD}$, considering two different applications.

\subsection{Case study 1: Rexnord/NASA data}

The first set of experimental data consists of the run-to-failure data of the Rexnord ZA-2115 double row bearings which were obtained by the NSF I/UCR Center for Intelligent Maintenance System with the support of Rexnord Corp. $(35,36)$. The rotation speed was set at a constant of $2000 \mathrm{rpm}$ and a radial load of approximately $26700 \mathrm{~N}$ was applied. The sampling rate was $20 \mathrm{kHz}$, with a one-second vibration signal being collected every 10 minutes. More details about the bearing and the test rig are in references $(35,36)$. The original data was sampled every 10 minutes but here the data are resampled 
every 30 minutes. Finally, 328 records are selected from the original 984 records to carry out the following analysis.

\subsection{Case study 2: Test rig for traction system of high-speed train}

Another set of data was collected during the endurance test on a full-scale test rig for high-speed train traction systems. The failure occurred on the bearing of the frame that was used to support the output shaft of the gearbox (Fig. 3). The damaged bearing is a cylindrical roller bearing (FAG NU1040M1) and the main damage, highlighted during the visual inspection after the bearing replacement, occurred in the inner race with many brinelling marks (Fig. 4). Even though the damaged bearing was an auxiliary component of the test rig, it was also monitored. The parameters of the bearing are shown in Table 1 . The rotation speed of the shaft was approximately $16.17 \mathrm{~Hz}$ and a constant torque was applied. The sampling rate was $20 \mathrm{kHz}$, and a five-second vibration signal was collected every hour. The experiment lasted for approximately 100 days and the system ran for approximately 8 hours every day. More details about the test rig and the experiment are in outlined in (37-39). Considering the effect of thermal expansion during the run-up from stand-still condition and the variation of environment condition, such as the room temperature, only four records from the second hour to the fifth hour are selected every day. Finally, 388 records are selected from the original 751 records for further analysis. It has been validated by many works in the literature that order tracking can mitigate the effect of speed variation when applied to bearing fault diagnosis. Since the tacho signal is available in this case study, order tracking is performed, and each record is resampled in angular domain, with 1226 samples in each revolution (40).

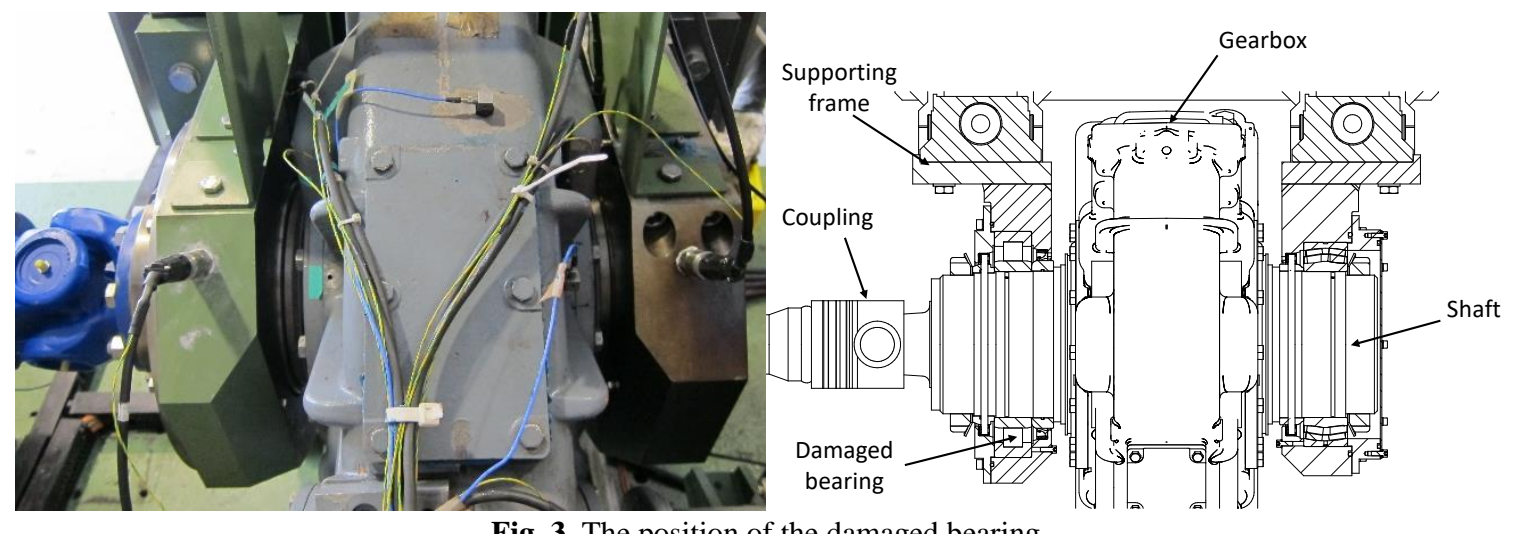

Fig. 3. The position of the damaged bearing

Table 1. The parameters of the tested bearing

\begin{tabular}{cccccc}
\hline Bearing code & Speed/[Hz] & BPFI/[NX] & BPFO/[NX] & FTF/[NX] & BSF/[NX] \\
\hline NU1040M1 & 16.17 & 13.20 & 10.80 & 0.45 & 4.85 \\
\hline
\end{tabular}

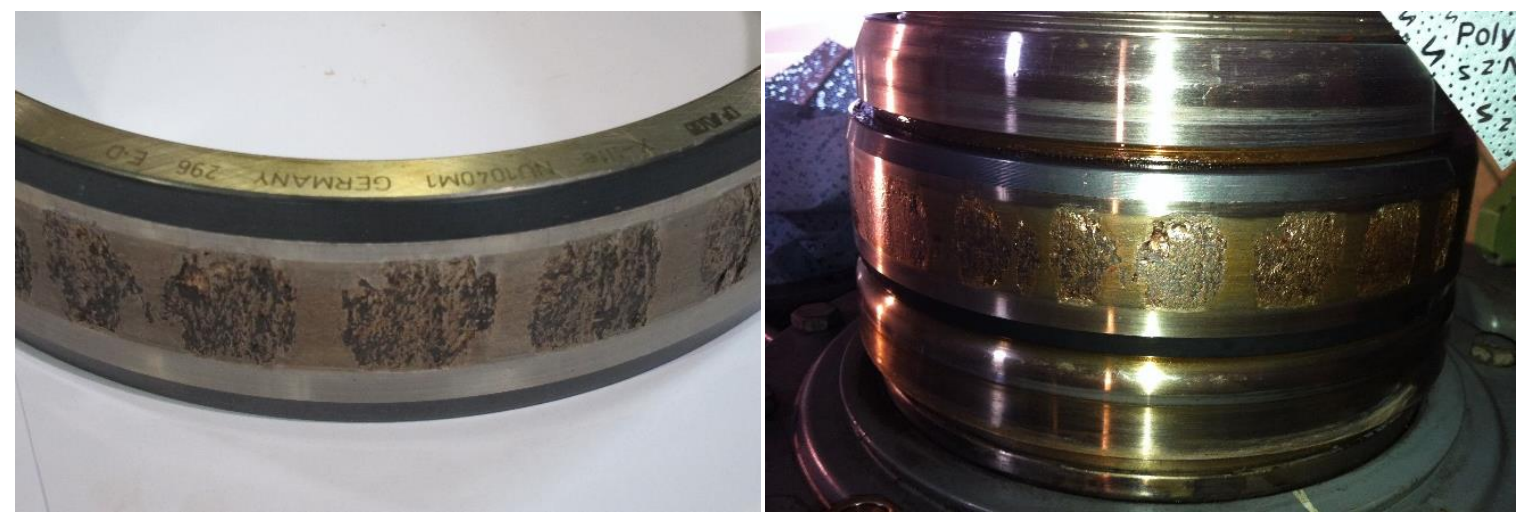

Fig. 4. The inter race of the damaged bearing of case study 2 


\subsection{Health state determination}

Assume that the evolution of the bearing health state is similar to that shown in Fig. 1(c), which would go through the previously mentioned five phases. The turning points and the rapidly drop points of the MAC ${ }^{2} P S D$ are seen as the potential candidates of boundary points for two HSs. Therefore, the MAC ${ }^{2}$ PSDs is used to divide the lifetime of a bearing into phases according to the variation trend of the MAC ${ }^{2} P S D$. The window size for the moving average operation clearly can affect the performance of the MAC ${ }^{2}$ PSD. The size of the window cannot be too large because it may produce too much smoothing in the MAC ${ }^{2} \mathrm{PSD}$, or too small because it may lead to heavy oscillations of the MAC ${ }^{2} \mathrm{PSD}$. An empirical suggestion is that the window size can be no more than $10 \%$ of the total number of records by considering the complete bearing life but not less than 8 .

Since the total numbers of records in the two sets of data are similar, the same window size will be used to calculate the MAC ${ }^{2}$ PSD. Three different window sizes, namely, 8, 16 and 24, are applied to obtain the MAC ${ }^{2}$ PSD for the two case studies. The corresponding results are displayed in Fig. 5. As expected, the MAC ${ }^{2}$ PSD of the two case studies with a window size of 8 (see Fig. 5(a), (b)) oscillates heavily and is unable to clearly distinguish different HSs. The MAC ${ }^{2} \mathrm{PSD}$ of the two case studies with a window size of 24 are shown in Fig. 5(e), (f) respectively, where the HSs of the two case studies cannot be clearly identified because the large window size smooths the MAC PSD too heavily. However, when a window size of 16 is applied (see Fig. 5(c), (d)), the MAC ${ }^{2}$ PSD of the two case studies clearly separate the different HSs, and increase steadily in phase \#4 as well. Therefore, a window size of 16 is used here for the two sets of data.

Conversely, the MAC ${ }^{2}$ PSDs of the two case studies do not have the same aspect as those of the schematic diagram shown in Fig. 1(b). However, it is possible to identify three main common characteristics. First, both of them have a clear steady-state phase (phase \#2) where the MAC ${ }^{2} P S D s$ fluctuate near a constant value (see Fig. 5(c), (d)); then, the MAC ${ }^{2}$ PSDs decrease like a step function after the steady state. Second, there are sharp drops between two consecutive states, such as from state 3 to 4 and from state 4 to 5 (see Fig. 5(c), (d)). Third, the most important is that both MAC PSDs increase smoothly in the defect propagation phase (phase \#4). However, there are also some differences between the two MAC ${ }^{2} P S D$. The MAC ${ }^{2} P S D$ of the first case study decreases from state 1 to state 2 (Fig. 5(c)), while the MAC ${ }^{2}$ PSD of the second case study increases from state 1 to state 2 (Fig. 5(d)). This is reasonable because the initial operating condition may be different. Furthermore, the MAC ${ }^{2}$ PSD of case study 1 clearly distinguishes states 2 and 3 (Fig. 5(c)), but it is not the same as that of the MAC ${ }^{2}$ PSD of case study 2 (Fig. 5(d)). This difference has been explained by the maintenance operations that occurred during the experimental activity, in which the gearbox was removed and reinstalled to perform visual inspection. This maintenance represents a discontinuity (circled in Fig. 5(d)) in the system behaviour and occurred exactly in correspondence of the transition from phase \#2 to \#3. About the RMS values of the two case studies, both remained almost constant until heavy damage occurred, especially the RMS value of the data from the high-speed train traction test rig only rapidly increases in the final phase (Fig. 5(d)). From the above analysis, it can be concluded that in comparison to the RMS value, the MAC ${ }^{2}$ PSD not only can clearly separate the HSs but can also clearly indicate the occurrence of defects earlier. 


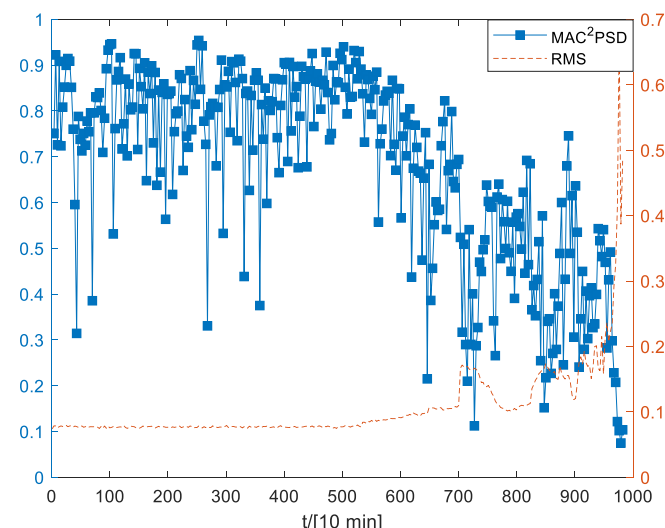

(a)

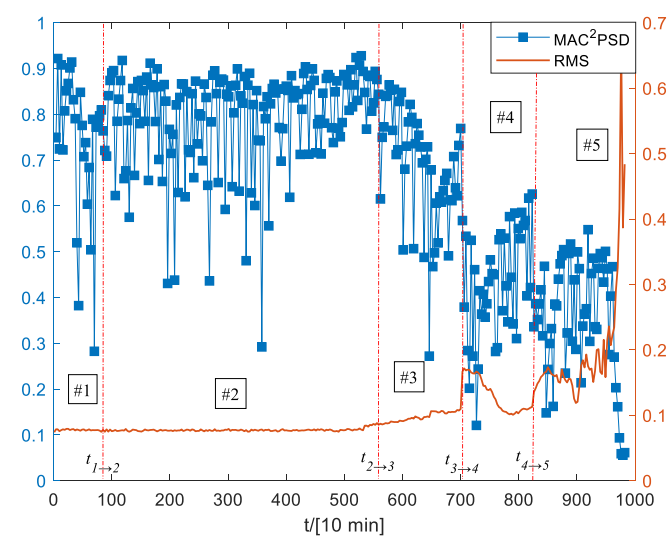

(c)

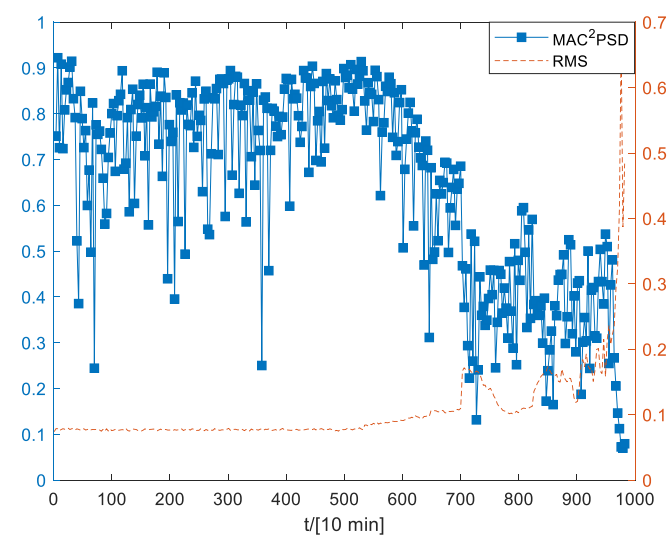

(e)

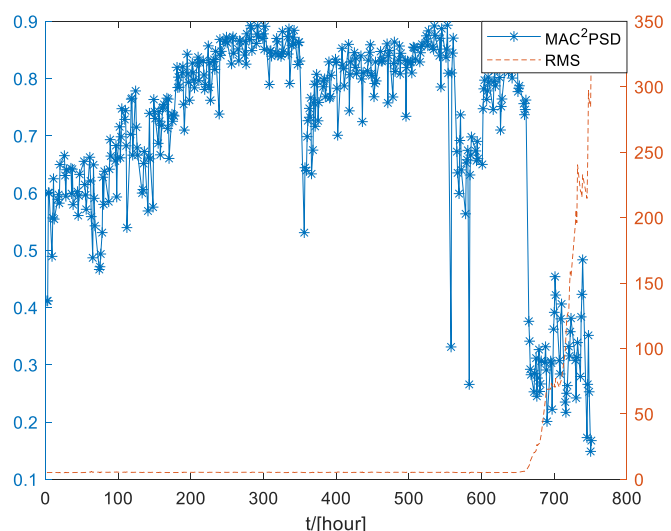

(b)

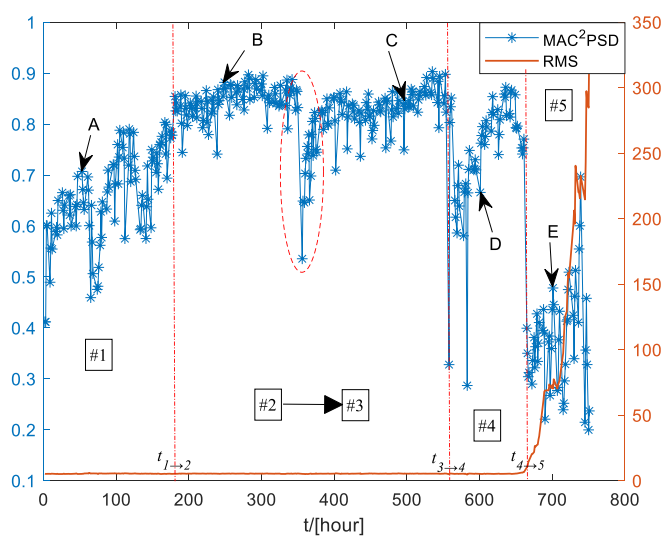

(d)

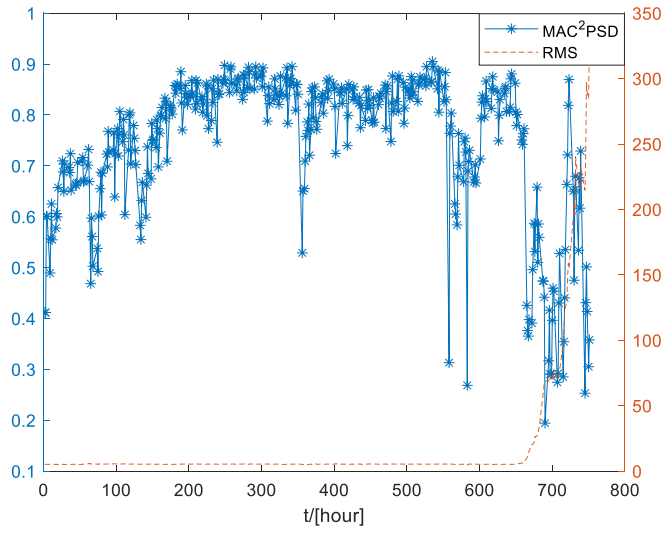

(f)

Fig. 5. $M A C^{2} P S D$ of actual vibration data; the $M A C^{2} P S D$ of case study 1 with time intervals of 8 (a), 16 (c) and 24 (e); the MAC ${ }^{2} P S D$ of case 2 with time intervals of 8 (b), 16 (d) and 24 (f).

To check the performance of the MAC ${ }^{2}$ PSD in the identification of HS phases, the health condition of the bearing has been checked at the five time instants A, B, C, D, E, (see Fig. 5(d)). The squared envelope spectra (SES) of the signals at the five time instants are displayed in Fig. 6. The optimal frequency bands used for the evaluation of each SES has been selected by PMFSgram (see (41)) in order domain and are listed in Table 2. Except for the last time instant E, the optimal frequency band of all the other four time instants are in the same narrow range of $180 \mathrm{NX}$ to $280 \mathrm{NX}$ and more or less overlap each other. It is clearly shown in Fig. 6 that all the SESs of the five points have a high value close to the BPFI or its second harmonic, which indicates the existence of defects on the inter race of the bearing. Although the amplitude of BPFI peaks increases slightly from time instant A to C (Fig. 6 (a), (b), (c)), it decreases in time instant D and it appears submerged at time instant E. Because of the complexity of the evolution of bearing damage, even though the SES method is suitable for detecting 
the damage on the bearing, it is not quite a suitable approach to monitoring the health condition of the bearing based directly on its amplitude.

Table 2. The optimal frequency band selected by the PMFSgram

\begin{tabular}{cccccc}
\hline Time instant & A & B & C & D & E \\
\hline Frequency band/[NX] & {$[187.7 \sim 247.1]$} & {$[215.8 \sim ~ 275.2]$} & {$[183.4 \sim 226.9]$} & {$[197.0 \sim 256.4]$} & {$[18.8 \sim 78.2]$} \\
\hline
\end{tabular}

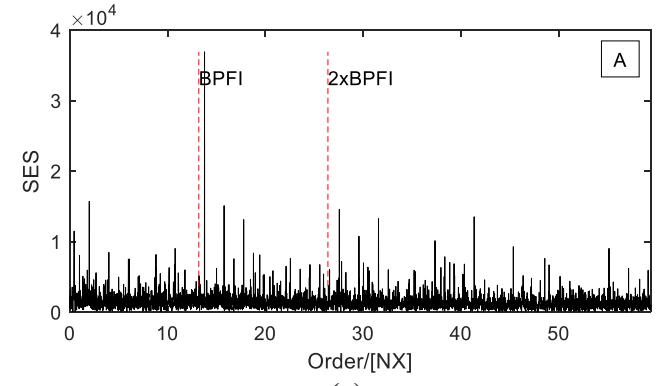

(a)

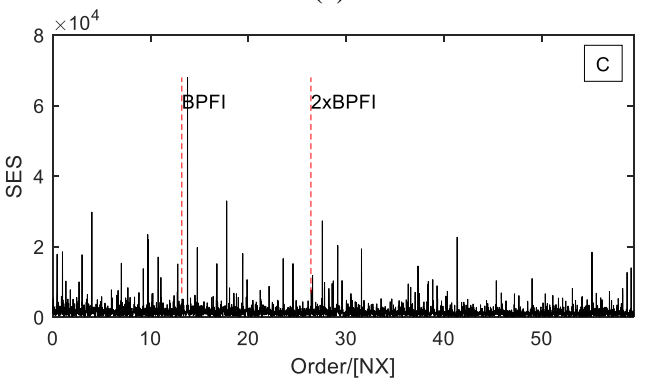

(c)

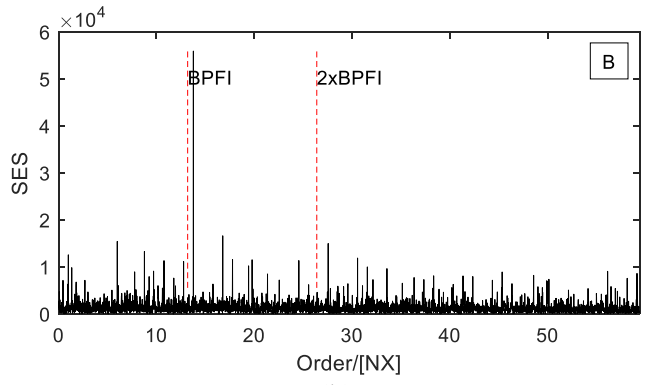

(b)

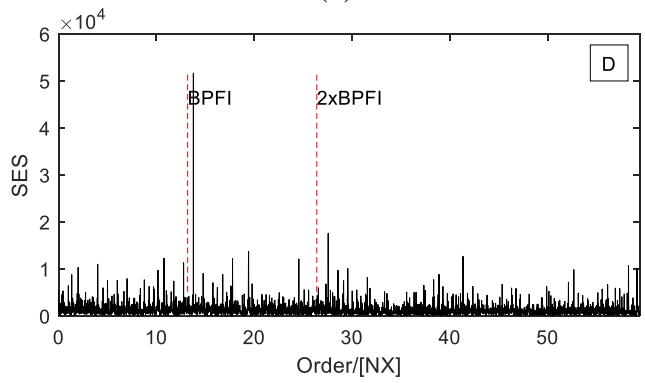

(d)

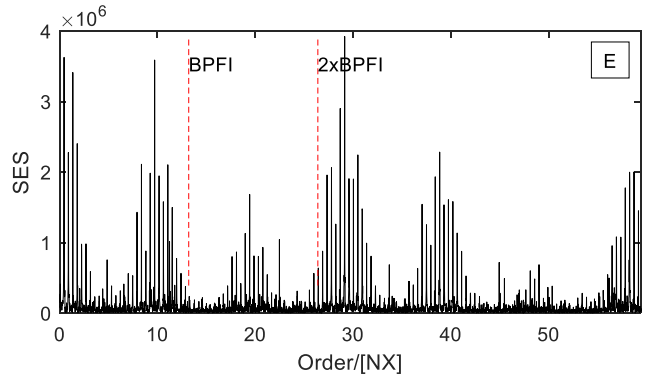

(e)

Fig. 6. The SES of the selected optimal frequency band; at time instant A (a), at time instant B (b), at time instant C (c), at time instant D (d), and at time instant $\mathrm{E}(\mathrm{e})$

\section{Estimation of the RUL of bearings based on MAC ${ }^{2}$ PSD}

The results obtained in the previous section have shown that the MAC ${ }^{2} \mathrm{PSD}$ is able to distinguish between the two last phases of the bearing life by means of a clear discontinuity. Thus, on one hand, it is meaningless to estimate the RUL of bearings using all the trends of the MAC PSD, but on the other hand, this property is convenient for setting the first prediction time. Only the MAC ${ }^{2} P S D$ values during the defect propagation phase (phase \# 4) are employed here, since the MAC ${ }^{2} P S D$ has a clear increasing trend in this phase. Compared with the phases of defect initiation (phase \#3) and damage growth (phase \#5), the increasing trend is noticeable during the defect propagation phase, and a mathematical model can be established.

\subsection{Determination of the threshold for the final failure}

In contrast to what occurs for most of the existing HIs, the determination of the threshold for the replacement before final failure for $\mathrm{MAC}{ }^{2} \mathrm{PSD}$, including a safety margin, is based on the 
characteristic of the defect development. The highest value of $\mathrm{MAC}^{2} \mathrm{PSD}$ is part of the defect initiation phase (phase \#3); this is characterized by an increasing trend and is used as a threshold value for the replacement. Fig. 7 shows how the threshold is defined for the two case studies described in section 3 .

The rationale of this choice lies in the property of MAC ${ }^{2}$ PSD that takes into account the similarity between the PSDs during the bearing life, i.e., the actual HS. Therefore, during the final phases of the bearing life, the MAC ${ }^{2}$ PSD should be less than the value it assumed after the defect onset. Similarly, during the last two phases, the highest value in each state should be less than that of the former state. Once the MAC ${ }^{2}$ PSD increases to the fixed threshold according to the trend of the defect propagation phase (phase \#4), the time $t_{\mathrm{EOL}}$ that corresponds to the end of life of the bearing can be estimated.

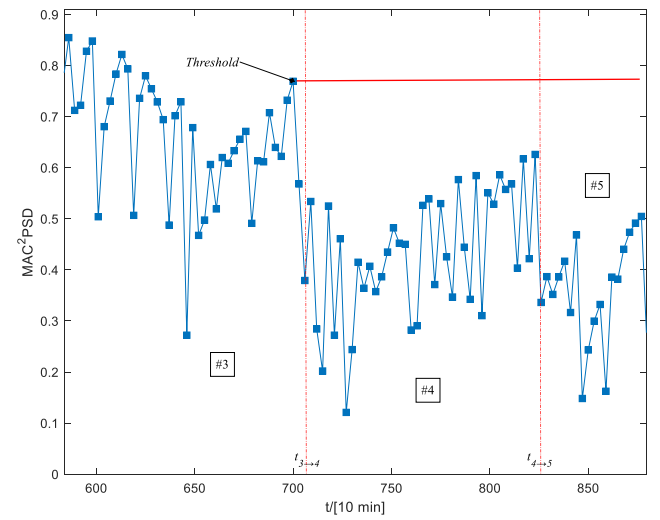

(a)

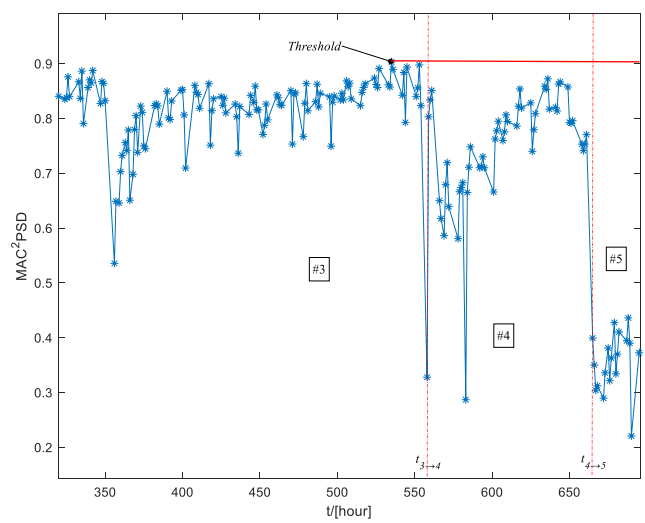

(b)

Fig. 7. The threshold of MAC ${ }^{2} P S D$; the threshold of MAC ${ }^{2} P S D$ for case study 1 (a) and the threshold of MAC PSD for case study 2 (b).

\subsection{Determination of the first predicting time}

The determination of the FPT is the first step for predicting the RUL. Since the trend of MAC PSD in the defect propagation phase (phase \#4) is almost monotonic, a linear model can be used to fit a set of MAC ${ }^{2} P S D$ data in phase \#4. The least squares fitting is used and the fitting line, at time $t_{3 \rightarrow 4}<t \leq t_{4 \rightarrow 5}$, is:

$$
y(t)=m_{t} t+q_{t}
$$

where $m_{t}$ is the slope and $q_{t}$ is the $y$-intercept, respectively, of the fitting line at time $t$.

The fitting operation starts after two records are obtained from the beginning of the defect propagation phase, i.e., $t \geq t_{(3 \rightarrow 4)+1}$. Considering the case study 2 of section 3, the different fitting lines and the corresponding slopes of the MAC ${ }^{2}$ PSD for an increasing set of data are shown in Fig. 8. Fig. 8(b) shows that the slope $m_{t}$ of $y(t)$ converges quickly by increasing the number of records used for the fitting to a rather stable value. However, the slope $m_{t}$ is below zero for several time instants before the time instant 593 in the magnified zone in Fig. 8(b). Considering the increasing trend of the MAC ${ }^{2} \mathrm{PSD}$ for the defect propagation phase (phase \#4), an additional constraint for the determination of the FPT is that the slope $m_{t}$ of the time instant of FPT should be positive. Similarly, Fig. 8(a) also shows that $y(t)$ fits the total trend of MAC ${ }^{2} \mathrm{PSD}$ with the increase in the number of records used in the fitting. 


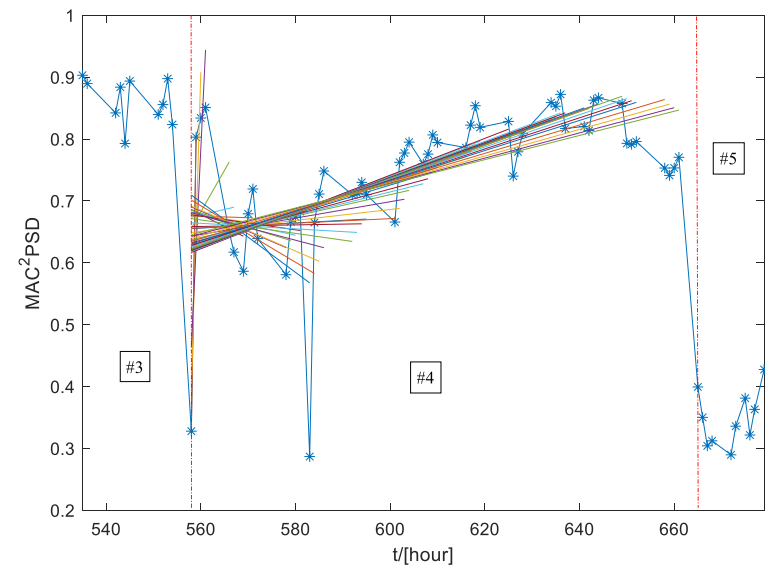

(a)

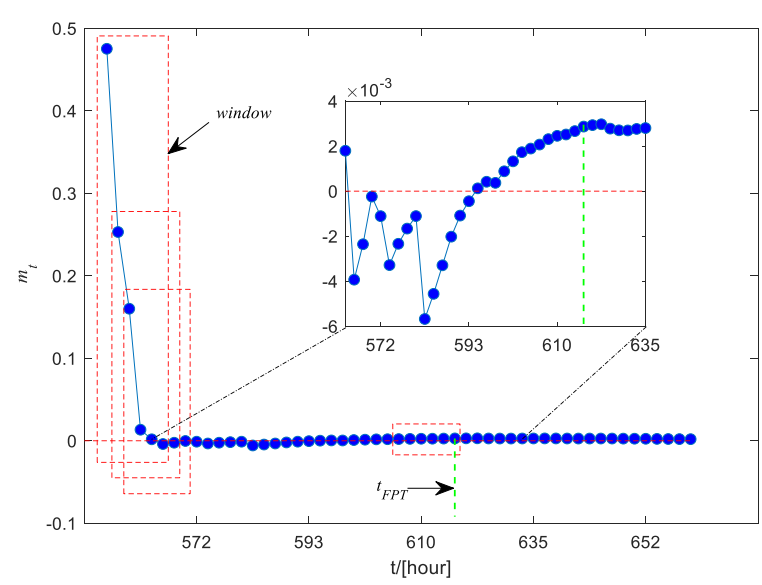

(b)

Fig. 8. The schematic diagram for determining the FPT; fitting lines in phase \#4 (a) and the slope of the fitting lines (b).

The criterion used in this paper to define the FPT is shown in Fig. 8(b); a moving-window-based method is applied to obtain the FPT, where the $t_{\mathrm{FPT}}$ is obtained as the first time instant for which either the relative rate of change of the mean value $\Delta \mu_{t}$ and variance $\Delta \sigma_{t}^{2}$ of the slope in the window are less than the given tolerance values $\varepsilon_{\mu}$ and $\varepsilon_{\sigma}$, respectively. The relative rate of change of the mean value $\Delta \mu_{t}$ and variance $\Delta \sigma_{t}^{2}$ of the slope in the window at time $t$ can be calculated as follows:

$$
\begin{gathered}
\Delta \mu_{t}=\left|\frac{\mu_{t}-\mu_{t-1}}{\mu_{t-1}}\right| \\
\Delta \sigma_{t}^{2}=\left|\frac{\sigma_{t}^{2}-\sigma_{t-1}^{2}}{\sigma_{t-1}^{2}}\right|
\end{gathered}
$$

where $\mu_{t}$ and $\mu_{t-1}$ are the mean values of the slope in the window at time $t$ and $t-1$ respectively, and $\sigma_{t}^{2}, \sigma_{t-1}^{2}$ are the variances of the slope in the window at time $t$ and $t-1$ respectively.

Therefore, at the first prediction time, $t_{\mathrm{FPT}}$, the following three conditions should be satisfied at the same time:

$$
\left\{\begin{array}{c}
\Delta \mu_{t_{F P T}} \leq \varepsilon_{\mu} \\
\Delta \sigma_{t_{F P T}}^{2} \leq \varepsilon_{\sigma} \\
m_{t_{F P T}}>0
\end{array}\right.
$$

where $\varepsilon_{\mu}, \varepsilon_{\sigma}$ are the given tolerance values for the relative rate of change of the mean value and variance respectively.

Since the slope $m_{t}$ converges quickly and the time span of phase \#4 is not very long, then a short window with a size of 6 is used here to determine the FPT. Meanwhile, the relatively small value of $10 \%$ is given for $\varepsilon_{\mu}$ and $\varepsilon_{\sigma}$. It is a relatively strong constraint for getting a reliable FPT because it means that from time instant $t_{F P T}-1$ to $t_{F P T}$, both $\Delta \mu_{t}$ and $\Delta \sigma_{t}^{2}$ change less than $10 \%$. The values of $\Delta \mu_{t}$ and $\Delta \sigma_{t}^{2}$ for the case study 2 are shown in Fig. 9, along with the threshold of $10 \%$ assumed for $\varepsilon_{\mu}$ and $\varepsilon_{\sigma}$. Finally, according to the above rules, the FPT of case study 1 is the time instant 772 and the FPT of case study 2 is the time instant of 618 . 


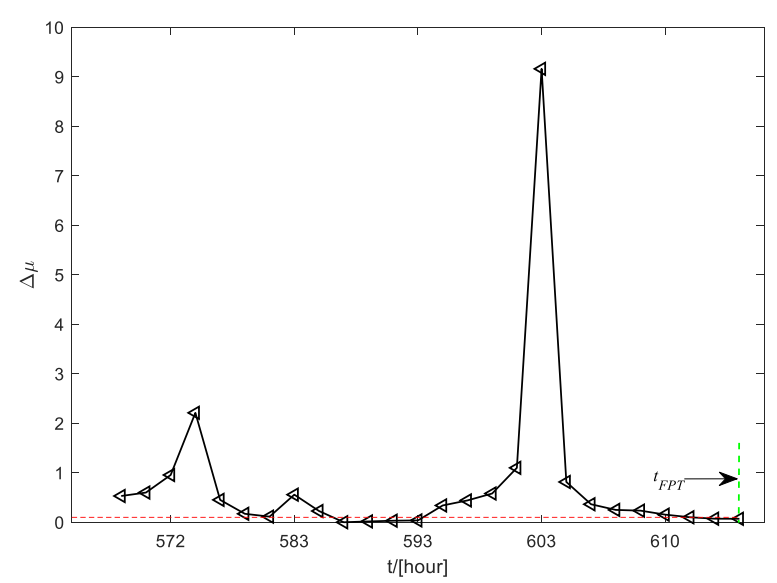

(a)

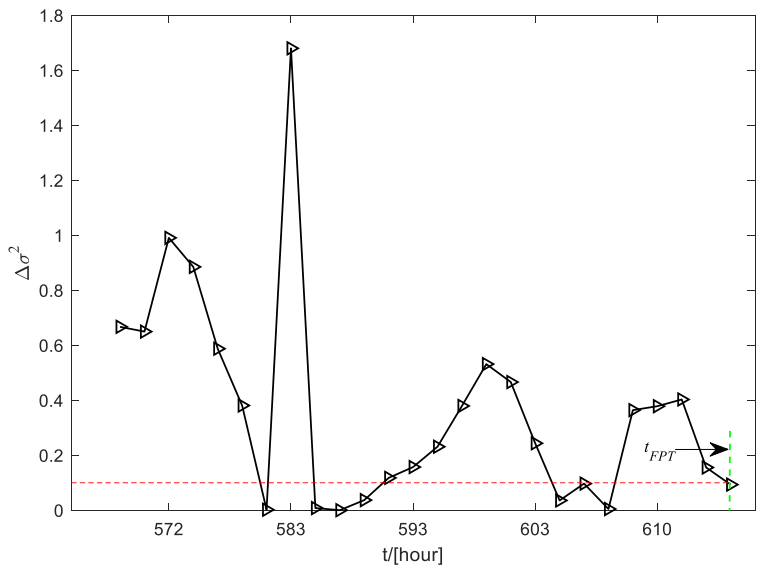

(b)

Fig. 9. The relative rate of change of the mean value and variance of the slope in the window for case study 2 ; the relative rate of change of the mean value (a) and the relative rate of change of the variance (b)

\subsection{Estimation of the RUL}

The estimation of the RUL starts after the FPT has been determined. If $m_{t}$ and $q_{t}$ are the slope and the $y$-intercept of the MAC ${ }^{2}$ PSD fitting line at time $t\left(t_{F P T} \leq t \leq t_{4 \rightarrow 5}\right)$, the final failure time of a bearing can be evaluated when the $y(t)$ equals the threshold (see Fig. 10). Hence, the estimation $\bar{t}_{\text {EOL }}$ of the end-of-life time of a bearing at time $t\left(t_{F P T} \leq t \leq t_{4 \rightarrow 5}\right)$ is given by:

$$
\bar{t}_{\mathrm{EOL}}(t)=\frac{\text { Threshold }-q_{t}}{m_{t}}
$$

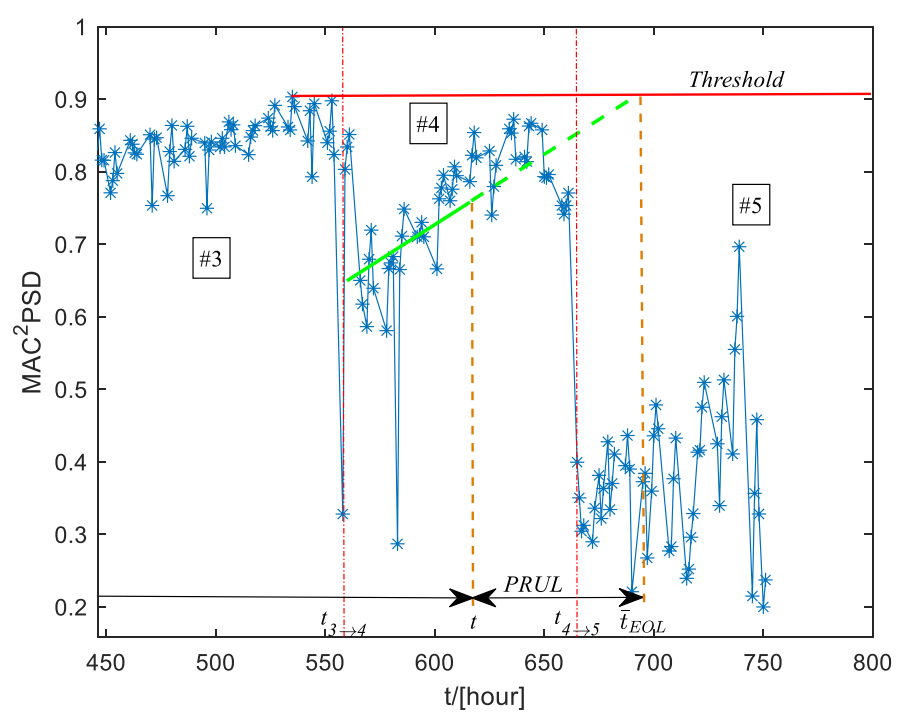

Fig. 10. The schematic diagram of estimating the RUL

The predicted RUL (PRUL) is the difference between the estimated end of lifetime and the time instant $t$, i.e.:

$$
\operatorname{PRUL}(t)=\bar{t}_{\mathrm{EOL}}(t)-t
$$

The actual RUL (ARUL), resulted from the run-to-failure tests, and the PRUL of the two case studies of section 3 are displayed in Fig. 11. It is clearly shown in Fig. 11(a) that the PRUL of case 1 oscillates near the ARUL, which means the result of the PRUL is reliable. However, in Fig. 11(b), the 
PRUL of case 2 oscillates below the ARUL, which means PRUL tends to zero slightly earlier than the ARUL. Furthermore, from the time instant $t_{\mathrm{FPT}}$ to the end of phase \#4 of time instant $t_{4 \rightarrow 5}$, a time span of the estimation of the end-of-life time $\bar{t}_{\text {EOL }}$ can also be obtained (see Fig. 11). The minimum estimation of the end-of-life time $\min \left(\bar{t}_{\mathrm{EOL}}\right)$ and the maximum estimation of the end-of-life time $\max \left(\bar{t}_{\mathrm{EOL}}\right)$ are the two limit values of $\bar{t}_{\mathrm{EOL}}$. The $\min \left(\bar{t}_{\mathrm{EOL}}\right)$ is suggested as the final time instant of end of life. Even though it may waste part of the useful lifetime of the bearing in an actual situation, the wasted lifetime is not far away from the time of the final failure. During the wasted lifetime, the bearing operation conditions are already very harsh. Therefore, the $\min \left(\bar{t}_{\mathrm{EOL}}\right)$ actually gives a further safety margin: the prediction of the failure time is slightly earlier than the actual failure time, which is very important for some expensive or high-accuracy mechanical systems. Hence, from the above two case studies, the estimation of RUL via MAC ${ }^{2}$ PSD is feasible and acceptable. The main shortcoming of MAC ${ }^{2}$ PSD applied to estimate the RUL is that the prediction time span could be short, but it could also be enough to assure the safe operation of machines, since the prediction time period is in the defect propagation phase but not in the final damage growth phase.

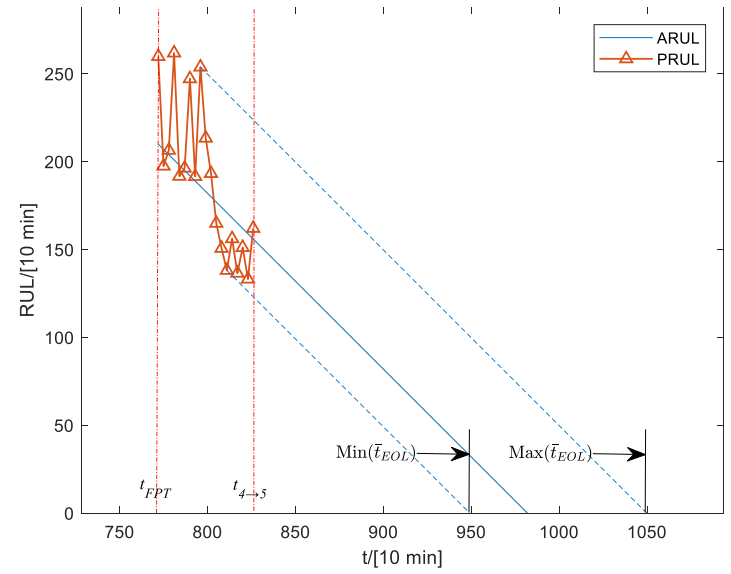

(a)

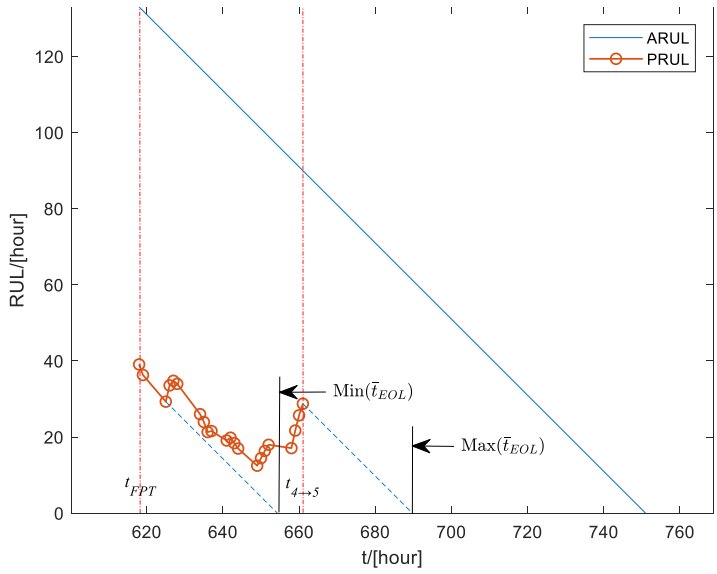

(b)

Fig. 11. The actual RUL and the prediction of RUL using MAC ${ }^{2} P S D$; the PRUL of bearing data from the NSF I/UCR Center (a) and the PRUL of bearing data from the high-speed train traction test rig (b).

\section{Capability of MAC ${ }^{2} P S D$ to avoid false positives}

Even though the effectiveness of MAC ${ }^{2}$ PSD has been proved using data from two different test rigs in Section 4, the capability of avoiding "false positive" detection in the bearing HS is evaluated by considering an even harsher operating condition, i.e., that of bearings installed in the traction system of a locomotive used for commercial service. Operating conditions are generally variable, including variation of speed, temperature, transmitted torque, track conditions, etc.

The locomotive used for the purpose of this research activity was placed in service in 2008 in the north of Italy, and a custom condition monitoring system was installed in late 2014. The sampling frequency is $25.6 \mathrm{kHz}$, and an eight-second signal is collected every two minutes. More details of the monitoring system are illustrated in (14). There was a scheduled overhaul from September 2015 and all the bearings on the locomotive powertrain were replaced by new bearings. Data from the end of 2014 to the end of 2017 of the motor bearing M1 of the unit 4 on the non-driven side are used to test the performance of MAC ${ }^{2}$ PSD. The bearing is an SKF 6318 deep-groove ball bearing. Outer race and cage damages (Fig. 12) on the bearing had been discovered by visual inspection during the overhaul at the end of 2015. Therefore, the conditions with a worn bearing (before the overhaul) and with a healthy new bearing (after the overhaul) can be considered. Even a large amount of data was collected, the operating conditions change all the time. Hence, before applying the MAC ${ }^{2} P S D$ method, the data 
need to be selected and filtered based on certain conditions, including the cruising speed between 140 $\mathrm{km} / \mathrm{h}$ to $150 \mathrm{~km} / \mathrm{h}$, the temperature of the bearing in the range of $70{ }^{\circ} \mathrm{C}$ to $100{ }^{\circ} \mathrm{C}$, the torque of the motor varying from $10 \%$ to $30 \%$ of the maximum torque, and the same rotation direction of the bearing. After the filtering operation, only 246 records and 734 records are selected before (worn bearing) and after (healthy new bearing) the overhaul, respectively.

An additional data checking process is performed, and some records are disregarded to assure that the final retained signals satisfy the following conditions: 1) the time difference of two consecutive records is larger than half an hour, 2) each record is quite stable during the sampling time window without an obvious jump from a certain time point, and 3) each record can carry out tacho-less order tracking correctly. A simple frequency domain tacho-less order tracking method is adopted here, which selects one clear harmonic of a reference signal, for example, the gear meshing signal, and then this harmonic is filtered out to go on the process of phase demodulation to obtain the phase information (42). Finally, 57 records and 175 records are kept, respectively, for the time before and after the overhaul. In an actual situation, the longer the time span, the harder it is for the locomotive to maintain a constant speed. Therefore, here only a one-second signal cut from the centre of the eightsecond signal is used for the following analysis. Since the number of records is comparatively small, a window with a size of 8 is used to obtain the MAC ${ }^{2} P S D$. The MAC ${ }^{2}$ PSD and RMS of those selected records are displayed in Fig. 13.

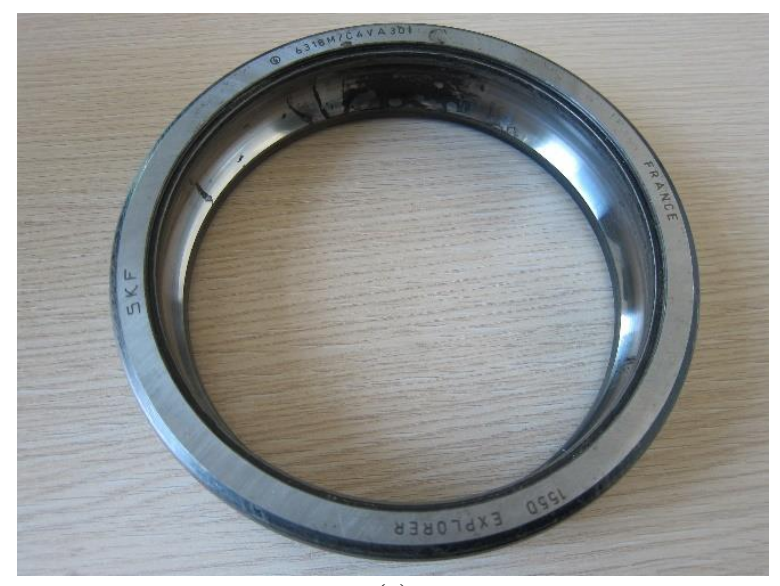

(a)

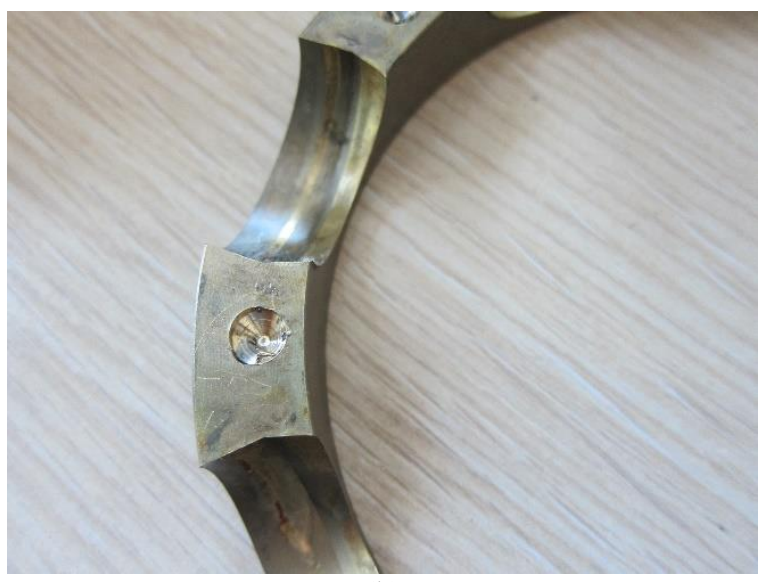

(b)

Fig. 12. Bearing damage inspection result; outer race damage (a) and cage damage (b).

As expected, the RMS shown in Fig. 13 cannot give any information indicating the health condition of the bearing. However, the MAC ${ }^{2}$ PSD has an obvious increasing trend before the time of overhaul (Fig. 13), which is quite similar to the increasing section of phase \#3 and \#4 of the two cases in section 3. This means that some defects may exist on the bearing and is in agreement with the inspection result of damages on the outer race and cage. Since the time span of the selected signal before the overhaul is only about half a year which is just about one-twelfth of the designed lifetime of the bearing and the bearing has been replaced before serious damage, it is impossible to confirm which phase the bearing was in, the defect initiation phase or the defect propagation phase. Compared to the MAC ${ }^{2}$ PSD before the overhaul, the MAC ${ }^{2} P S D$ after the overhaul generally oscillates but assumes high values in the range of 0.8 to 1 , which means the bearing is in good condition (see for instance the trend of Fig. 4 in phase \#2). This is accords well with the fact that a new bearing was installed after the overhaul. Furthermore, there is no obvious transition of the MAC2PSD at the beginning after a new bearing was installed, which may be because the running-in state is very short and without apparent differences compared with the steady state. Even though an obvious drop (circled in Fig. 13) occurs in the MAC ${ }^{2}$ PSD close to the end of Nov. 2016, there is no obvious difference in MAC $^{2}$ PSD before and after that time instant. It can be seen as a normal fluctuation. 
Although the operating conditions of the bearing on the commercial locomotive are very complex, the MAC ${ }^{2} P S D$ is still effective, and its performance is very stable.

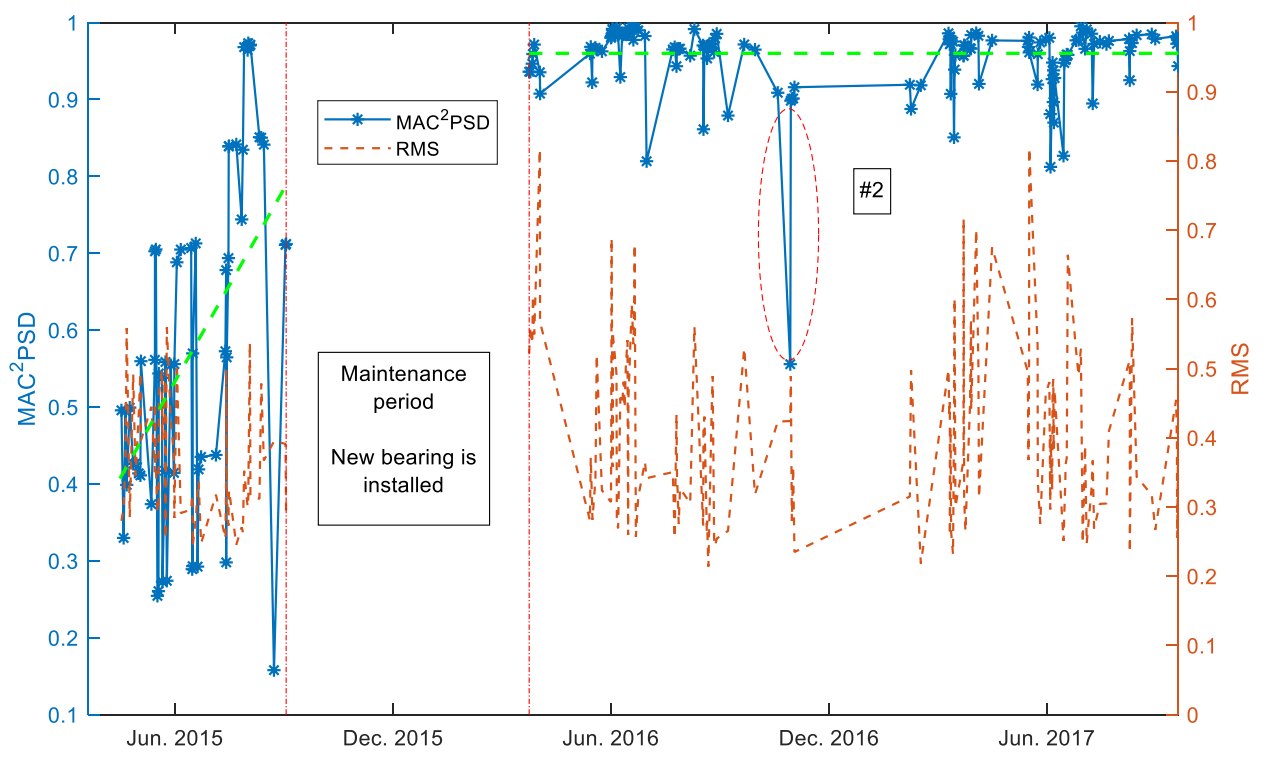

Fig. 13. The MACPSD of bearing data from the locomotive

\section{Conclusions}

In this paper, to realize the goal of bearing CM, a new HI called 'MAC ${ }^{2} P S D$ ' is proposed based on the moving average cross-correlation of the PSD of the signals. The MAC ${ }^{2} P S D$ method utilizes the relationship between the variation in PSD and the development of the defects in bearings and is able to track the evolution of health condition and can clearly divide the HSs of bearings. In comparison with RMS, MAC ${ }^{2} P S D$ can indicate the occurrence of a defect earlier and more clearly. Furthermore, a linear model is proposed to estimate the RUL of bearings according to the trend of the MAC PSD in the defect propagation phase. Compared with most of the existing health indices, the MAC ${ }^{2}$ PSD can determine the failure threshold by itself. The MAC PSD performs well when applied to two sets of bearing vibration data from two different test rigs. In addition, the effectiveness of MAC ${ }^{2}$ PSD in avoiding false positives is tested via actual bearing vibration data from a commercial locomotive. MAC ${ }^{2}$ PSD is a useful HI for bearing CM. However, MAC ${ }^{2}$ PSD can only estimate the RUL in the propagation phase. Extension of the prediction time span and means of dealing with the gap between the PRUL and ARUL will be discussed in the future.

\section{References}

1. SKF Group. Bearing damage and failure analysis. SKF Group; 2017. p. 101.

2. Loutas TH, Roulias D, Georgoulas G. Remaining useful life estimation in rolling bearings utilizing data-driven probabilistic Esupport vectors regression. IEEE Trans Reliab. 2013;

3. Lei Y, Li N, Guo L, Li N, Yan T, Lin J. Machinery health prognostics: A systematic review from data acquisition to RUL prediction. Mech Syst Signal Process [Internet]. 2018;104:799-834. Available from: http://www.sciencedirect.com/science/article/pii/S0888327017305988

4. El-Thalji I, Jantunen E. A summary of fault modelling and predictive health monitoring of rolling element bearings. Mech Syst Signal Process [Internet]. 2015;60-61:252-72. Available from: http://www.sciencedirect.com/science/article/pii/S0888327015000813

5. Sikorska JZ, Hodkiewicz M, Ma L. Prognostic modelling options for remaining useful life estimation by industry. Mech Syst Signal Process [Internet]. 2011;25(5):1803-36. Available from: http://www.sciencedirect.com/science/article/pii/S0888327010004218

6. Heng A, Tan ACC, Mathew J, Montgomery N, Banjevic D, Jardine AKS. Intelligent condition-based prediction of machinery reliability. Mech Syst Signal Process [Internet]. 2009;23(5):1600-14. Available from: 
http://www.sciencedirect.com/science/article/pii/S088832700900003X

7. Zhang B, Zhang L, Xu J. Degradation Feature Selection for Remaining Useful Life Prediction of Rolling Element Bearings. Qual Reliab Eng Int [Internet]. 2016 Mar 1;32(2):547-54. Available from: https://doi.org/10.1002/qre.1771

8. Li N, Lei Y, Lin J, Ding SX. An Improved Exponential Model for Predicting Remaining Useful Life of Rolling Element Bearings. IEEE Trans Ind Electron. 2015;

9. Javed K, Gouriveau R, Zerhouni N, Nectoux P. A feature extraction procedure based on trigonometric functions and cumulative descriptors to enhance prognostics modeling. In: PHM 2013 - 2013 IEEE International Conference on Prognostics and Health Management, Conference Proceedings. 2013.

10. Lu C, Chen J, Hong R, Feng Y, Li Y. Degradation trend estimation of slewing bearing based on LSSVM model. Mech Syst Signa Process [Internet]. 2016;76-77:353-66. Available from: http://www.sciencedirect.com/science/article/pii/S0888327016000820

11. El-Thalji I, Jantunen E. Dynamic modelling of wear evolution in rolling bearings. Tribol Int [Internet]. 2015;84:90-9. Available from: http://www.sciencedirect.com/science/article/pii/S0301679X1400423X

12. El-Thalji I, Jantunen E. Fault analysis of the wear fault development in rolling bearings. Eng Fail Anal [Internet]. 2015;57:470-82 Available from: http://www.sciencedirect.com/science/article/pii/S1350630715300522

13. Lei Y, Li N, Lin J. A New Method Based on Stochastic Process Models for Machine Remaining Useful Life Prediction. IEEE Trans Instrum Meas. 2016;

14. Pennacchi P, Chatterton S, Vania A, Xu L. Diagnostics of Bearings in Rolling Stocks: Results of Long Lasting Tests for a Regional Train Locomotive. In: Mechanisms and Machine Science. 2019.

15. Borghesani P, Pennacchi P, Chatterton S. The relationship between kurtosis- and envelope-based indexes for the diagnostic of rolling element bearings. Mech Syst Signal Process. 2014;43(1-2):25-43.

16. Ocak H, Loparo KA, Discenzo FM. Online tracking of bearing wear using wavelet packet decomposition and probabilistic modeling: A method for bearing prognostics. J Sound Vib [Internet]. 2007;302(4):951-61. Available from: http://www.sciencedirect.com/science/article/pii/S0022460X07000260

17. Ben Ali J, Chebel-Morello B, Saidi L, Malinowski S, Fnaiech F. Accurate bearing remaining useful life prediction based on Weibull distribution and artificial neural network. Mech Syst Signal Process [Internet]. 2015;56-57:150-72. Available from: http://www.sciencedirect.com/science/article/pii/S0888327014004087

18. Huang HZ, Wang HK, Li YF, Zhang L, Liu Z. Support vector machine based estimation of remaining useful life: current research status and future trends. J Mech Sci Technol. 2015;

19. Hong S, Zhou Z, Lu C, Wang B, Zhao T. Bearing remaining life prediction using Gaussian process regression with composite kernel functions. J Vibroengineering. 2015;17(2):695-704.

20. Medjaher K, Zerhouni N, Baklouti J. Data-driven prognostics based on health indicator construction: Application to PRONOSTIA's data. In: 2013 European Control Conference (ECC). 2013. p. 1451-6.

21. Yu J. Adaptive hidden Markov model-based online learning framework for bearing faulty detection and performance degradation monitoring. Mech Syst Signal Process [Internet]. 2017;83:149-62. Available from: http://www.sciencedirect.com/science/article/pii/S0888327016301674

22. Guo L, Li N, Jia F, Lei Y, Lin J. A recurrent neural network based health indicator for remaining useful life prediction of bearings Neurocomputing [Internet]. 2017;240:98-109. Available from: http://www.sciencedirect.com/science/article/pii/S0925231217303363

23. El-Thalji I, Jantunen E. A descriptive model of wear evolution in rolling bearings. Eng Fail Anal [Internet]. 2014 Oct 1 [cited 2019 Apr 30];45:204-24. Available from: https://www.sciencedirect.com/science/article/pii/S1350630714001885

24. Randall RB. Vibration-Based Condition Monitoring: Industrial, Aerospace and Automotive Applications. Vibration-Based Condition Monitoring: Industrial, Aerospace and Automotive Applications. 2010.

25. Antoni J. Cyclostationarity by examples. Mech Syst Signal Process [Internet]. 2009;23(4):987-1036. Available from: http://www.sciencedirect.com/science/article/pii/S0888327008002690

26. Lanza A, Morigi S, Sgallari F, Yezzi AJ. Variational Image Denoising Based on Autocorrelation Whiteness. SIAM J Imaging Sci. 2013;

27. Borghesani P, Ricci R, Chatterton S, Pennacchi P. A new procedure for using envelope analysis for rolling element bearing diagnostics in variable operating conditions. Mech Syst Signal Process. 2013;38(1).

28. Baraldi P, Cannarile F, Di Maio F, Zio E. Hierarchical k-nearest neighbours classification and binary differential evolution for fault diagnostics of automotive bearings operating under variable conditions. Eng Appl Artif Intell [Internet]. 2016 Nov 1 [cited 2019 Apr 29];56:1-13. Available from: https://www.sciencedirect.com/science/article/pii/S0952197616301427?via\%3Dihub

29. Stefaniak PK, Zimroz R, Sliwinski P, Andrzejewski M, Wyłomanska A. Multidimensional signal analysis for technical condition, operation and performance understanding of heavy duty mining machines. In: Applied Condition Monitoring. 2016. 
stationarity - A procedure of load-dependent features processing with application to wind turbine bearings. Mech Syst Signal Process [Internet]. 2014 May 3 [cited 2019 Apr 29];46(1):16-27. Available from:

https://www.sciencedirect.com/science/article/pii/S0888327013004974?via\%3Dihub

31. Dao PB, Staszewski WJ, Barszcz T, Uhl T. Condition monitoring and fault detection in wind turbines based on cointegration analysis of SCADA data. Renew Energy [Internet]. 2018;116:107-22. Available from: http://www.sciencedirect.com/science/article/pii/S0960148117305931

32. Viadero F, Fernández A, Iglesias M, de-Juan A, Liaño E, Serna MA. Non-stationary dynamic analysis of a wind turbine power drivetrain: Offshore considerations. Appl Acoust [Internet]. 2014 Mar 1 [cited 2019 Apr 30];77:204-11. Available from: https://www.sciencedirect.com/science/article/pii/S0003682X13002417?via\%3Dihub

33. Saxena A, Celaya J, Balaban E, Goebel K, Saha B, Saha S, et al. Metrics for evaluating performance of prognostic techniques. In 2008 International Conference on Prognostics and Health Management, PHM 2008. 2008.

34. Lei Y. Intelligent Fault Diagnosis and Remaining Useful Life Prediction in Rotating MAchinery. Butterworth-Heinemann; 2017. $1-366 \mathrm{p}$.

35. Qiu H, Lee J, Lin J, Yu G. Wavelet filter-based weak signature detection method and its application on rolling element bearing prognostics. J Sound Vib [Internet]. 2006;289(4):1066-90. Available from: http://www.sciencedirect.com/science/article/pii/S0022460X0500221X

36. Lee J, Qiu H, Yu G, Lin J. Rexnord Technical Services (2007).'Bearing Data Set', IMS, University of Cincinnati. NASA Ames Prognostics Data Repository. 2009.

37. Chatterton S, Borghesani P, Pennacchi P, Vania A. Optimal Frequency Band Selection for the Square Envelope Spectrum in the Diagnostics of Rolling Element Bearings. In: Proceedings of the ASME Design Engineering Technical Conference. 2015. p V008T11A003.

38. Pennacchi P, Bruni S, Chatterton S, Borghesani P, Ricci R, Marinis D, et al. A Test Rig for the Condition-Based Maintenance Application on the Traction Chain of Very High Speed Trains. In: World Congress on Railway Research. 2011.

39. Chatterton S, Borghesani P, Pennacchi PEL, Vania AT. Experimental Evidences in the Monitoring of Rolling Element Bearings. J Vib Eng Technol. 2016;4(5):395-402.

40. Fyfe KR, Munck EDS. ANALYSIS OF COMPUTED ORDER TRACKING. Mech Syst Signal Process. 2002;

41. Xu L, Chatterton S, Pennacchi P. A Novel Method of Frequency Band Selection for Squared Envelope Analysis for Fault Diagnosing of Rolling Element Bearings in a Locomotive Powertrain. Vol. 18, Sensors . 2018.

42. Coats M, Sawalhi N, Randall RB. Extraction of tacho information from a vibration signal for improved synchronous averaging. Proc Acoust. 2009;

\section{Acronyms}

\begin{tabular}{|c|c|}
\hline ANN & Artificial Neural Network \\
\hline ARUL & Actual Remaining Useful Life \\
\hline BPFI & Ballpass frequency, inner race \\
\hline BPFO & Ballpass frequency, outer race \\
\hline $\mathrm{BSF}$ & Ball (roller) spin frequency \\
\hline $\mathrm{CM}$ & Condition Monitoring \\
\hline FTF & Fundamental train frequency (cage speed) \\
\hline FPT & First Prediction Time \\
\hline GPR & Gaussian Process Regression \\
\hline $\mathrm{HI}$ & Health Index \\
\hline HMM & Hidden Markov Model \\
\hline HS & Health State \\
\hline RUL & Remaining Useful Life \\
\hline $\mathrm{MAC}^{2}$ & Moving Average Cross-Correlation \\
\hline $\mathrm{MAC}^{2} \mathrm{PSD}$ & Moving Average Cross-Correlation of the Power Spectral Density \\
\hline PCA & Principle Component Analysis \\
\hline PCC & Pearson's Correlation Coefficient \\
\hline PSD & Power Spectral Density \\
\hline PRUL & Predicted Remaining Useful Life \\
\hline $\mathrm{RCC}$ & Cyclic Content Ratio \\
\hline REB & Rolling Elements Bearing \\
\hline RNN & Recurrent Neural Network \\
\hline RMS & Root Mean Square \\
\hline
\end{tabular}


SES Squared Envelope Spectrum

SVM Support Vector Machine 